\title{
Fatty acid-binding proteins in Echinococcus spp.: the family has grown
}

\author{
Jorge L. Pórfido ${ }^{1,2}$ - Michaela Herz ${ }^{3}$. Ferenc Kiss ${ }^{3}$ - Laura Kamenetzky ${ }^{4} \cdot$ Klaus Brehm $^{3} \cdot$ Mara C. Rosenzvit $^{4}$. \\ Betina Córsico ${ }^{1} \cdot$ Gisela R. Franchini ${ }^{1}$ (I)
}

Received: 8 August 2019 / Accepted: 18 February 2020

(C) Springer-Verlag GmbH Germany, part of Springer Nature 2020

\begin{abstract}
Fatty acid-binding proteins (FABPs) are small intracellular proteins that reversibly bind fatty acids and other hydrophobic ligands. In cestodes, due to their inability to synthesise fatty acids de novo, FABPs have been proposed as essential proteins, and thus, as possible drug targets and/or carriers against these parasites. We performed data mining in Echinococcus multilocularis and Echinococcus granulosus genomes in order to test whether this family of proteins is more complex than previously reported. By exploring the genomes of E. multilocularis and E. granulosus, six genes coding for FABPs were found in each organism. In the case of E. granulosus, all of them have different coding sequences, whereas in E. multilocularis, two of the genes code for the same protein. Remarkably, one of the genes (in both cestodes) encodes a FABP with a C-terminal extension unusual for this family of proteins. The newly described genes present variations in their structure in comparison with previously described FABP genes in Echinococcus spp. The coding sequences for $E$. multilocularis were validated by cloning and sequencing. Moreover, differential expression patterns of FABPs were observed at different stages of the life cycle of E. multilocularis by exploring transcriptomic data from several sources. In summary, FABP family in cestodes is far more complex than previously thought and includes new members that seem to be only present in flatworms.
\end{abstract}

Keywords Echinococcus multilocularis $\cdot$ FABPs $\cdot$ Fatty acids $\cdot$ Cestodes

\section{Introduction}

Echinococcosis is caused by the metacestode larval stages of Echinococcus spp., which generate cyst-like structures in the intermediate host's inner organs. Cystic and alveolar

Section Editor: Bruno Gottstein

Gisela R. Franchini

gfranchini@ conicet.gov.ar

1 Instituto de Investigaciones Bioquímicas de La Plata (INIBIOLP), Facultad de Ciencias Médicas, Universidad Nacional de La Plata (UNLP)-Consejo Nacional de Investigaciones Científicas y Técnicas (CONICET), La Plata, Argentina

2 Present address: Institut Pasteur Montevideo, Montevideo, Uruguay

3 Institut für Hygiene und Mikrobiologie, Universität Würzburg, Würzburg, Germany

4 Instituto de Investigaciones en Microbiología y Parasitología Médica (IMPaM), Facultad de Medicina, Universidad de Buenos Aires (UBA)-Consejo Nacional de Investigaciones Científicas y Técnicas (CONICET), Buenos Aires, Argentina echinococcosis, produced by Echinococcus granulosus sensu lato and Echinococcus multilocularis, respectively, affect more than one million people around the world. These diseases can significantly reduce life quality and can be life threatening if untreated. The main treatments consist on either surgery or prolonged drug therapy, with varying results (World Health Organization 2019).

Due to the inability of cestodes to synthesise fatty acids de novo (Maule and Marks 2006; Smyth and McManus 2007), fatty acid-binding proteins (FABPs), together with other lipidbinding proteins, have been proposed as essential for these organisms, facilitating lipid uptake from the host. These proteins have also been suggested as possible antiparasitic drug targets and/or carriers (Xu et al. 2011).

FABPs are small intracellular proteins (around $15 \mathrm{kDa}$ ) that reversibly bind fatty acids and other hydrophobic ligands. Members of this family have been found throughout the animal kingdom, both in vertebrates and invertebrates, but no counterparts have been found in plants or fungi (Haunerland and Spener 2004). They are usually highly expressed in cells with very active lipid metabolism. The sequence identity 
among the family varies significantly but they share an overall similar 3D protein structure consisting in a solvent accessible $\beta$-barrel with an alpha helical cap. In E. granulosus, two FABPs have been described, EgFABP1 and EgFABP2 (Esteves et al. 1993, 2003). EgFABP1 has been characterised in more detail, including its 3D structure clearly matching the typical structure of FABPs (Jakobsson et al. 2003), binding properties as well as its interaction with ligands and with artificial lipidic membranes (Alvite et al. 2001; Pórfido et al. 2012).

This study offers a detailed analysis of the FABP family in E. multilocularis and E. granulosus not only based on genomic and transcriptomic information but also validated by cloning and sequencing of the respective cDNAs from $E$. multilocularis.

\section{Results and discussion}

We identified six FABP encoding genes on the available E. multilocularis genome. Two of them encode proteins with identical primary sequence and $93 \%$ identity to EgFABP1; thus, they were named emfabp1.1 [EmuJ_002165500] and emfabp1.2 [EmuJ_000550000] (accession codes correspond to WormBase ParaSite). Of the other four genes, one is orthologous to egfabp2 [EmuJ_000549800] (100\% identity between EmFABP2 and EgFABP2), whereas the other three represent novel genes (named emfabp3 [EmuJ_000551000], emfabp4 [EmuJ_000417200] and emfabp5 [manually annotated by us], each with distinctive features. In particular, although the coding sequence of emfabp3 is slightly longer than previously described for egfabpl and egfabp2, the gene contains a considerable longer intron (4443 versus 80 bp) reminiscent of those from mammalian FABPs (Esteves and Ehrlich 2006; Alvite et al. 2008; Smathers and Petersen 2011) (Fig. 1). emfabp4, on the other hand, codes for a longer protein compared with all other FABPs (176 versus 130 aminoacids), and also has an unusually long intron compared with what had been mostly described for cestodes FABPs (Esteves et al. 2003; Esteves and Ehrlich 2006; Alvite et al. 2008). In this work, the coding sequences abovementioned have been corroborated by cloning and sequencing of E. multilocularis cDNAs. Obtained sequences have been deposited in Gene Bank under the following accession codes: MN809107 (emfabp 1.2); MN809108 (emfabp 1.1); MN809109 (emfabp2); MN809110 (emfabp3); MN809111 (emfabp4); MN809112 (emfabp5). It is important to note that Zheng and co-workers (2013b) have proposed the presence of more than two FABP genes in Echinococcus spp. In particular, based on in silico data, the authors stated the existence of five genes each in E. granulosus and E. multilocularis. Importantly, our study revealed several differences to those predictions (Zheng et al. 2013b). On the one hand, these authors proposed intronless genes that would correspond to

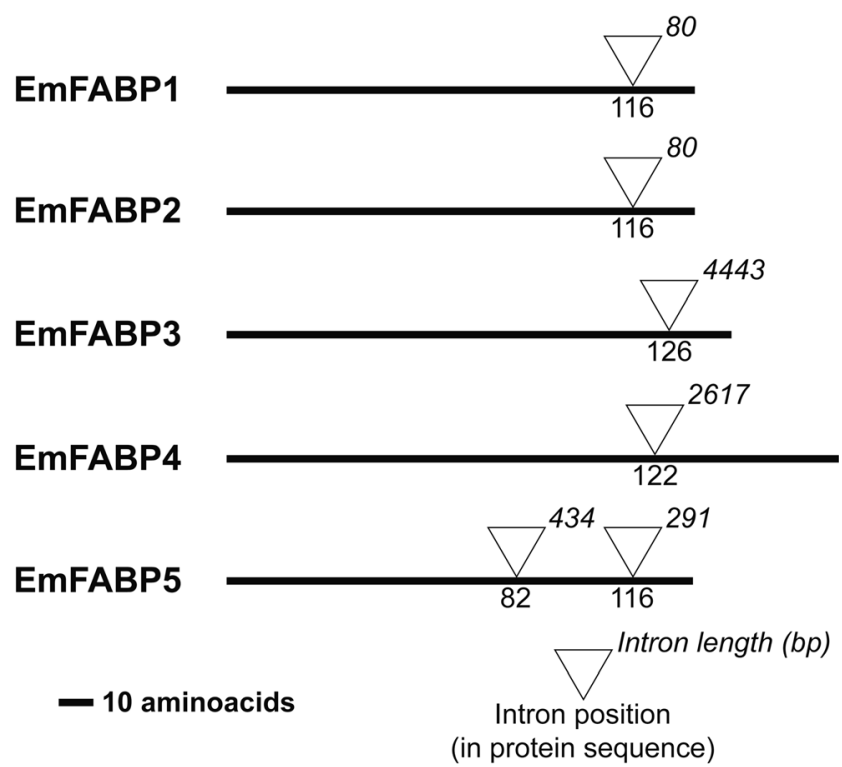

Fig. 1 Schematic representation of the length and position of the introns in EmFABPs' protein sequences. The figure displays the length of the introns (in base pairs) at the upper-right corner of the triangles as well as the relative position of the splicing sites in the translated protein sequence for the five EmFABPs (under the triangles). EmFABP1 accounts for EmFABP1.1 and EmFABP1.2 since they are identical

emfabp3 and emfabp4, but that does not agree with our experimental evidence (cloning and sequencing of $E$. multilocularis cDNAs). We found, instead, that both these genes have unusually long introns compared with what had been described for cestodes FABPs (Esteves et al. 2003; Esteves and Ehrlich 2006; Alvite et al. 2008). In addition to that, the protein sequences proposed by them for EmFABP3 and EmFABP4 (called Emul_FABP1 and Emul_FABP4, respectively, in their article) are identical to our proposed EmFABP3 and EmFABP4 up to the single intron splice site in each gene. After that site, their Emul_FABP1 continues with amino acids that do not share similarities with equivalent FABPs' positions, and Emul_FABP4 ends at amino acid 124. Finally, we found that emfabp5 was similar to emfabp1 and emfabp2, although carrying two introns instead of the only one previously described in cestodes FABP genes (Esteves and Ehrlich 2006; Alvite et al. 2008). More than one intron is not uncommon for FABP genes of different species, but it has not been described in cestodes yet (Zimmerman and Veerkamp 2002; Esteves and Ehrlich 2006; Smathers and Petersen 2011). Moreover, in intron 1 of emfabp5, an alternative GC splice donor sequence was found which, although being less common than GT, appears quite frequently in species such as Homo sapiens, Caenorhabditis elegans and Arabidopsis thaliana (Sheth et al. 2006; Parada et al. 2014).

With respect to genomic organization, five of the genes (emfabp1.1, emfabp1.2, emfabp2, emfabp3 and emfabp4) are present on the same chromosome (chromosome 7), and all of them but emfabp3 are coded by the same strand. The other 
gene, emfabp5, lies on chromosome 4. In addition, three of the genes encoded by chromosome 7 (emfabp1.1, emfabp 1.2 and emfabp2) are clustered in E. multilocularis within a $13.7-\mathrm{kb}$ region, with intergenic regions of $5.6 \mathrm{~kb}$ (between emfabp2 and emfabp 1.1) and $6.7 \mathrm{~kb}$ (between emfabp 1.1 and emfabp1.2).

The six predicted protein sequences of FABPs were assigned to the FABP family according to different patterns found in their primary structure: PROSITE pattern Cytosolic fatty-acid binding proteins signature (accession number PS00214), Pfam pattern Lipocalin/Cytosolic fatty acid binding protein family (accession number PF00061) and PRINTS pattern Fatty acid-binding protein signature (accession number PR00178). The PRINTS pattern is defined by 3 elements. The first one includes the first $\beta$-strand ( $\beta A$ ) that forms part of the barrel and the first $\alpha$-helix $(\alpha \mathrm{I})$. Additionally, on $\beta \mathrm{A}$, it has a characteristic Gly-X-Trp triplet (where X represents any amino acid). The second element goes from the $\mathrm{C}$-terminal end of $\beta \mathrm{D}$ across $\beta \mathrm{E}$, and the third represents the last two strands of the barrel, $\beta \mathrm{I}$ and $\beta \mathrm{J}$. Within these three elements, all six FABPs are very well conserved (Fig. 2). What is more, they all invariantly contain the P2 ligand-binding motif (an Arg... Arg-x-Tyr motif involved in ligand binding) (Jones et al. 1988; Jakobsson et al. 2003), with the sole exception of EmFABP4 that has a Tyr instead of an Arg in the first position.
All the analysed sequences showed a predicted secondary structure of ten $\beta$-strands with two $\alpha$-helices between the first two strands, in good accordance with the typical arrangement of secondary structure elements in FABPs. In the case of EmFABP3, the prediction includes longer turns between strands $\beta B$ and $\beta C$, and between $\beta F$ and $\beta G$. Nevertheless, whether the insertions affect these turns or other regions of the protein remains to be determined. Finally, in EmFABP4, the most striking feature is that the predicted sequence is much longer (176 amino acids) than what is expected for a FABP (around 130 amino acids). In this case, a typical FABP fold is predicted but no specific structure is assigned to the $\mathrm{C}$ terminus of the protein. The coding sequence has been amplified by RT-PCR, which gives evidence for its correct transcription and expression within the organism so it is expected to constitute a new FABP variant in which the $\mathrm{C}$ terminus' fold and function should be addressed. It is noticeable that, recently, FABPs with C-terminal extensions from Fasciola spp. have been also reported (Morphew et al. 2016).

In order to define the relatedness of the proteins across the species, a phylogenetic analysis was performed using FABPs from cestodes and model organisms (Fig. 3). As observed, all cestodes FABPs are clustered together with the sole exception of EmFABP5 and EgFABP5 which represent a different node. This protein sequence divergence is in accordance with its
Fig. 2 Multiple alignment of protein sequences of EmFABPs. This figure shows the alignment of the EmFABPs. Three regions are highlighted with dotted lines, representing the three elements that constitute PRINTS pattern PR00178 (fatty acid-binding protein signature) which represent a fingerprint of the FABP family. The grey-shaded amino acids represent conserved positions whereas light blue-shaded ones represent positions with similar amino acids in the different proteins. (*) indicates the P2-binding motif. The yellow bars represent approximate location of $\beta$-sheets $(\beta \mathrm{A}-\beta \mathrm{J})$, while red bars symbolise $\alpha$-helices ( $\alpha \mathrm{I}$ and $\alpha \mathrm{II})$, both according to EgFABP1 structure (PDB, 1o8v) and secondary structure predictions performed employing PSIPRED tool

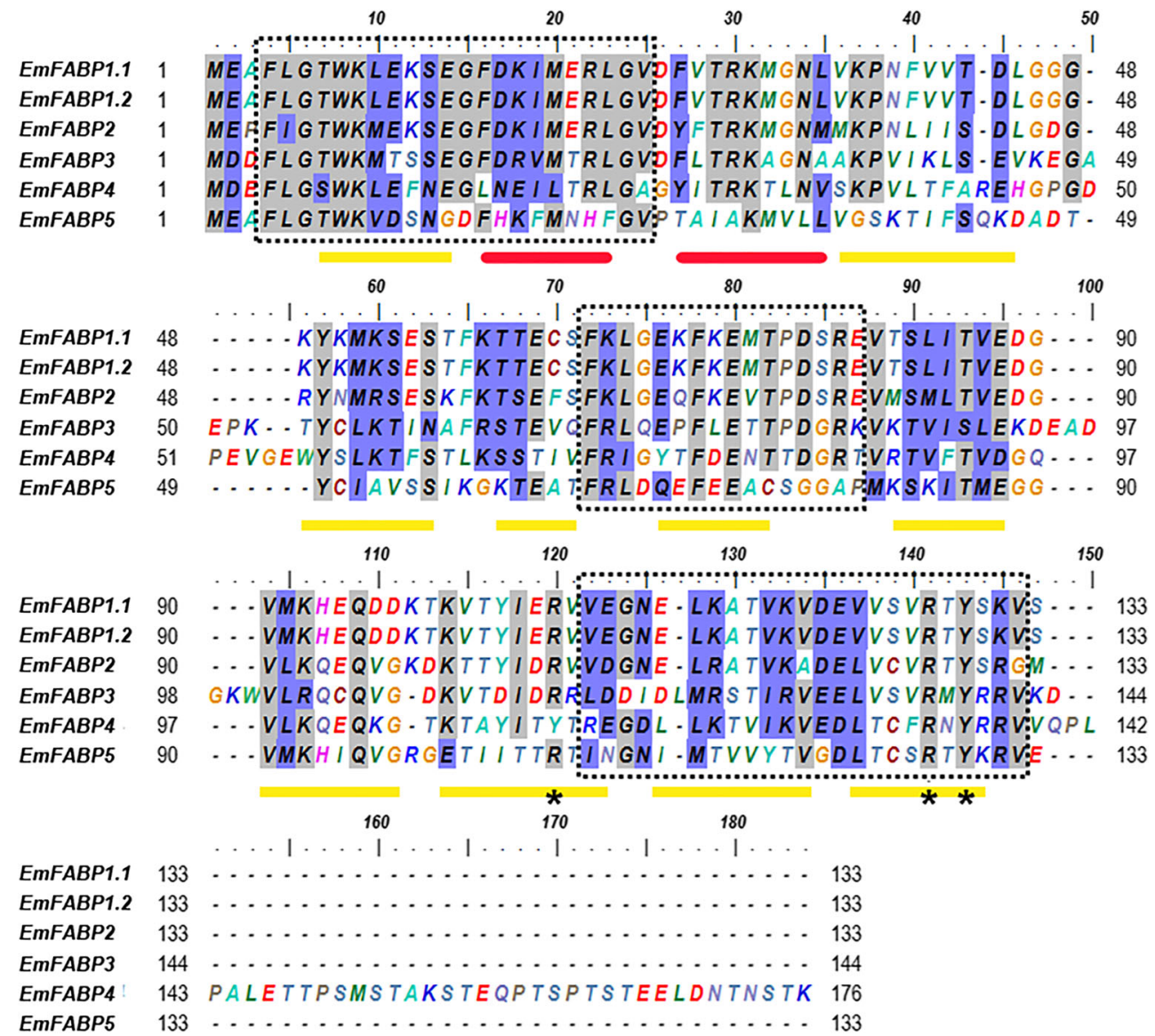




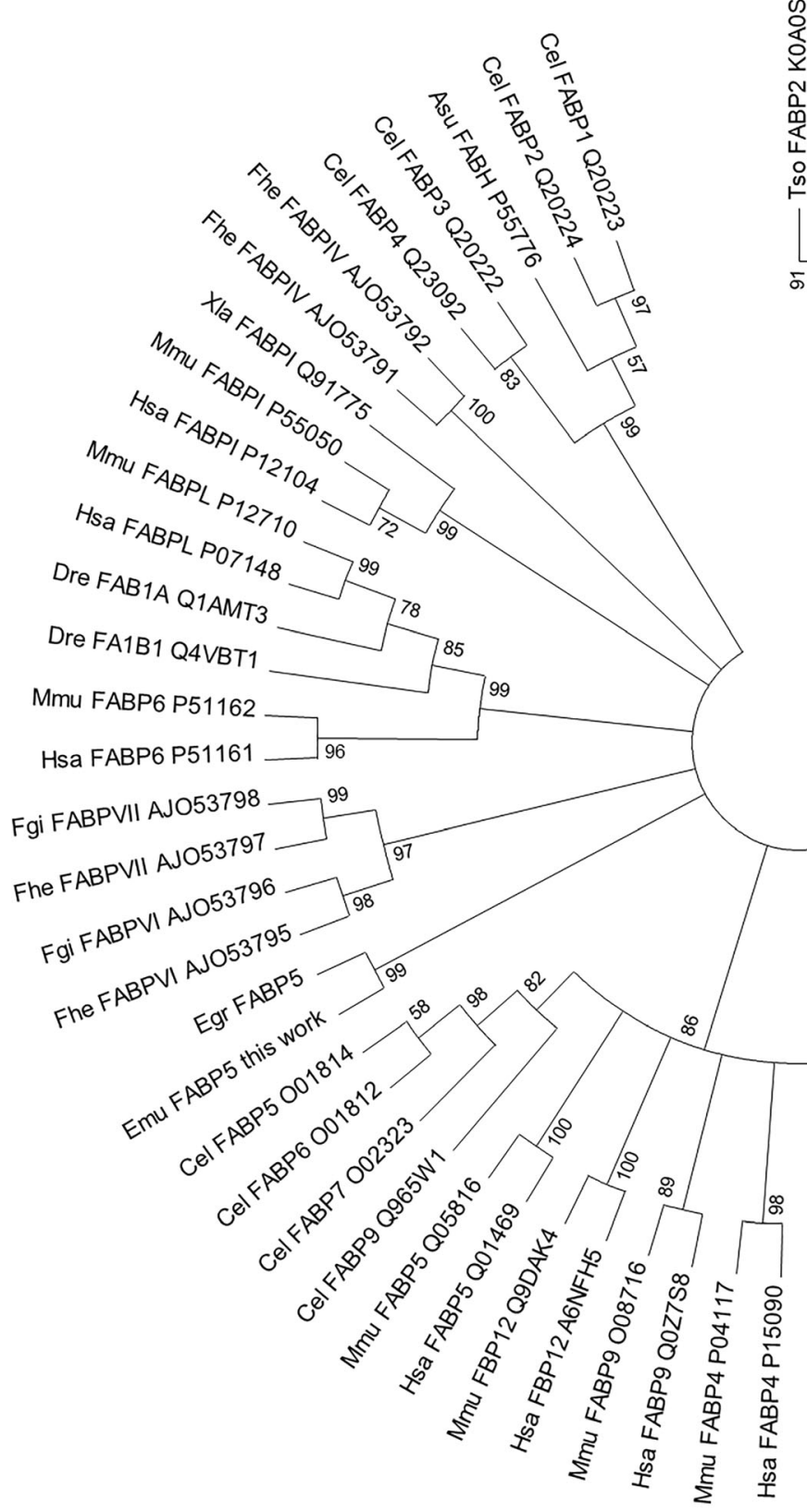

Fig. 3 Phylogenetic relationships of FABPs from different Phyla. Phylogenetic tree of the amino acid sequences of the FABPs among the following species: Hsa Homo sapiens, Mmu Mus musculus, Fhe Fasciola hepatica, Egr Echinococcus granulosus, Emu Echinococcus multilocularis, Cel Caenorhabditis elegans, Dre Danio rerio, Hta Hydatigera taeniformis, Xla Xenopus laevis, Asu Ascaris suum, Csi Clonorchis sinensis, Sja Schistosoma japonicum, Sma Schistosoma mansoni, Fgi Fasciola gigantica, Hna Hymenolepis nana, Hdi Hymenolepis diminuta, Tso Taenia solium, Tas Taenia asiatica, Tmu Taenia multiceps, Tpi Taenia pisciformis. Gene IDs are shown next to

differential genetic structure since EmFABP5 and EgFABP5 carry two introns instead of the only one previously described in cestodes FABP genes, as previously mentioned (Esteves and Ehrlich 2006; Alvite et al. 2008).

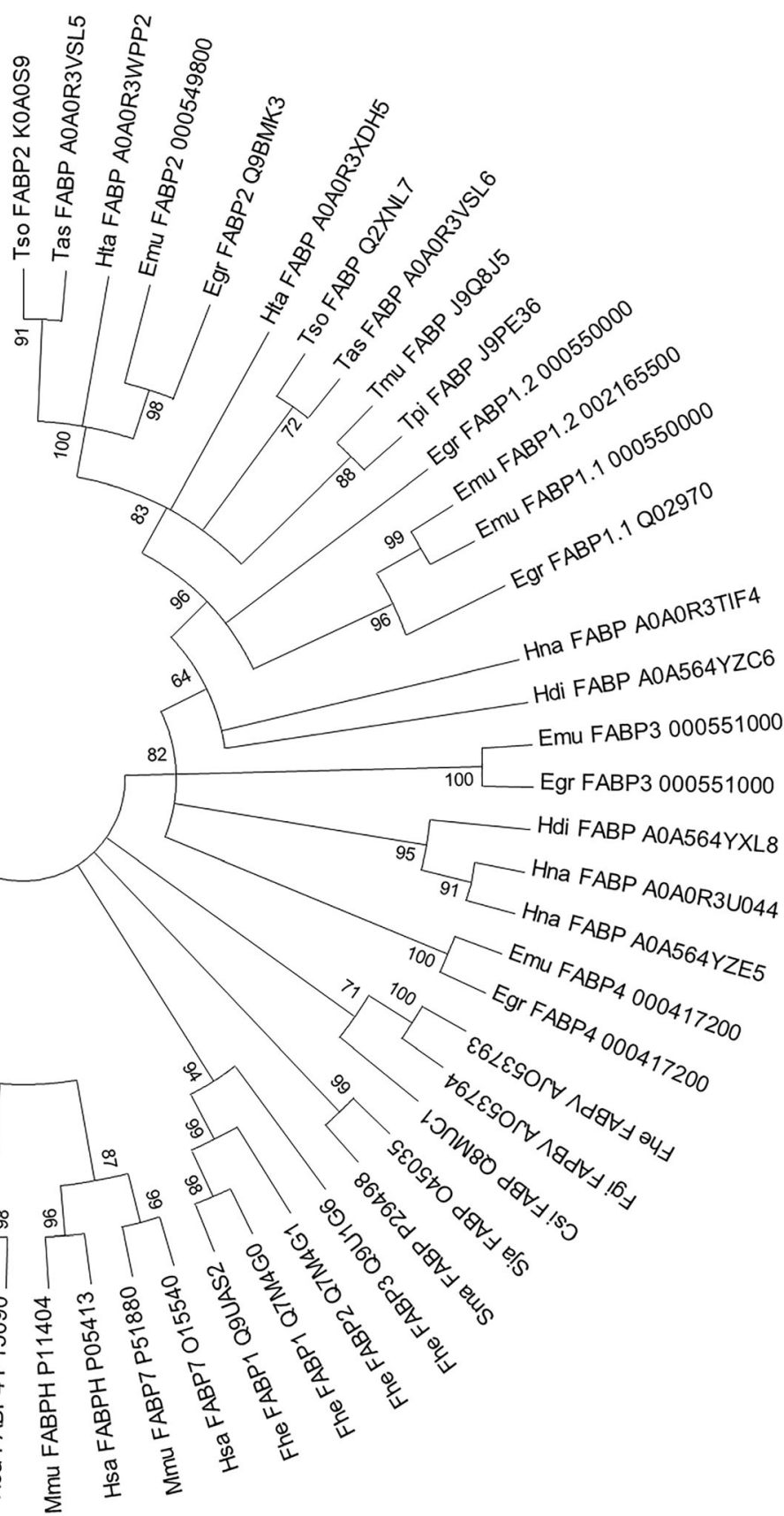

the proteins' common name. Phylogenetic tree was obtained using the maximum likelihood method based on the JTT matrix-based model. Branches corresponding to partitions reproduced in less than $50 \%$ bootstrap replicates are collapsed, and the percentage of replicate trees in which the associated taxa clustered together in the bootstrap test (500 replicates) are shown next to the branches. The analysis involved 90 amino acid sequences. All positions with less than $95 \%$ site coverage were eliminated. There were a total of 79 positions in the final dataset. Phylogenetic analysis was conducted in the MEGA7

After analysing the FABP genes in the genome of E. multilocularis, the available genome of E. granulosus was also explored, employing the obtained EmFABPs sequences as queries. As expected, six genes coding for 
FABPs were found in E. granulosus as well. Two of them correspond to the previously reported egfabpl (from now on named egfabp 1.1) [EgrG_000549850 commented on database] and egfabp2 [EgrG_000549800] (Esteves et al. 1993, 2003). Three other genes are, clearly, orthologues of emfabp 3, emfabp 4 and emfabp5, and thus named egfabp 3 [EgrG_000551000], egfabp4 [EgrG_000417200] and egfabp 5 [EGR_05655, commented on database]. The sixth gene turned out to be located in a cluster equivalent to that described above for E. multilocularis. It is found immediately after egfabp2 and egfabp1.1, but in this case, the CDS (or the ORF) is not identical to that of egfabp1.1. As it is placed in an equivalent position to where emfabp1.2 is found in the E. multilocularis genome, relative to other FABP genes, it was named egfabpl.2 [EgrG_000550000].

Noticeable is the fact that in EgFABP1.2, two of the three amino acids that shape the P2-binding motif are not conserved. This could imply that this protein may bind its ligands employing a different mechanism (as certain mammal FABPs that lack this motif do), bind other kind of ligands or be nonfunctional.

Recently published transcriptomic information regarding E. multilocularis oncospheres together with transcriptomic data published by Tsai and co-workers (2013) suggest that all the genes analysed in the present work (with the exception of emfabp 5 that was not annotated) are transcribed in different stages of E. multilocularis (Zheng et al. 2013a; Huang et al. 2016).

In addition to that, the transcriptomic data published later, together with the genome of E. granulosus (Zheng et al. 2013a), indicate that all the FABP genes of E. granulosus are transcribed in, at least, one stage of the parasite (egfabpl.1 and egfabp 5 are not well annotated in that genome).

According to the different sets of data, emfabp1.1 and emfabp 1.2 are the most highly expressed FABP genes in E. multilocularis (data not shown). Given that emfabp1.1 and emfabp1.2 are mostly identical in CDS sequence, it is hard to distinguish which reads correspond to each of the two genes. However, the flanking regions of both genes present several differences that allowed us to map transcripts unequivocally to the genome, reinforcing the existence of two genes (emfabp1.1 and emfabp1.2) and giving evidence that both are transcribed and show differences in expression profile (Fig. 4).

Although expressed in many stages, emfabp 2 is reported to show higher expression levels in metacestodes and adult stage, as well as in primary cells (i.e., cells isolated from metacestode tissues of Echinococcus multilocularis which are able to regenerate vesicles in vitro, part of them being germinal cells, the only proliferative ones (Koziol et al. 2014)). emfabp 3 seems to be poorly expressed, being higher at the adult stage. Moreover, reported data indicates that it would be expressed at the oncosphere stage (non-activated) of E. multilocularis, which are indeed included in the gravid adult samples employed for the analysis (Huang et al. 2016). In the case of emfabp 4, it is more expressed in adult stages, both gravid and pre-gravid (Tsai et al. 2013). A reanalysis of the data obtained by Tsai et al. showed that emfabp 5 is moderately expressed with its expression higher in the late metacestode stage, which contains brood capsules with protoscoleces. In the adult stage, it would be expressed in levels equivalent to emfabp3 (Fig. 4).

In the case of E. granulosus, according to Zheng et al. (2013a), egfabp1.2 is more highly transcribed in adult stage than in oncosphere, protoscoleces or cyst, suggesting a possible expression in adult tissues absent in the other stages. egfabp1.1 is very highly expressed in all stages, egfabp 2 mainly in adult and cyst, egfabp 3 in oncospheres and cyst, and egfabp 4 primarily in adults (Zheng et al. 2013a). egfabp5, which is not properly annotated in Zheng et al. (2013a) but, however, is present in transcriptomic data, could be confined to oncospheres and adult stage (which could be due to the expression in oncospheres contained in the adult, as previously mentioned).

It is worth noticing that, in every case, each stage is considered as a whole, not having into account (for technical reasons) the different tissues that compose them. It is thus important, in the future, to deepen the study of those aspects of FABPs expression.

This study shows that FABP family in cestodes is far more complex than previously thought and includes new members, some of them with features that could be unique to flatworms.

\section{Materials and methods}

\section{Bioinformatic analysis}

The high-quality E. multilocularis genome assembly and E. granulosus sensu stricto draft genome assembly (Tsai et al., 2013) were retrieved from the WormBase Parasite database (http://parasite.wormbase.org/). The reference sequences EgFABP1 (GenBank AF321119.1) and EgFABP2 (GenBank AF321117.1) from E. granulosus were used to perform BLASTN, BLASTP, TBLASTN and BLASTX (hosted in the sequence alignment editor BioEdit, http:// www.mbio.ncsu.edu/bioedit/bioedit.html) against $E$. multilocularis and E. granulosus genomes. The predicted protein sequences were analysed employing InterPro (http:// www.ebi.ac.uk/interpro/), for domain identification, and PSIPRED (http://bioinf.cs.ucl.ac.uk/psipred/), for secondary structure prediction.

In order to detect possible expression of all E. multilocularis FABPs, including emfabp1.1, emfabp1.2 and emfabp 5, two approaches were used. In the first approach, RNAseq data from (Tsai et al. 2013) was remapped to the 

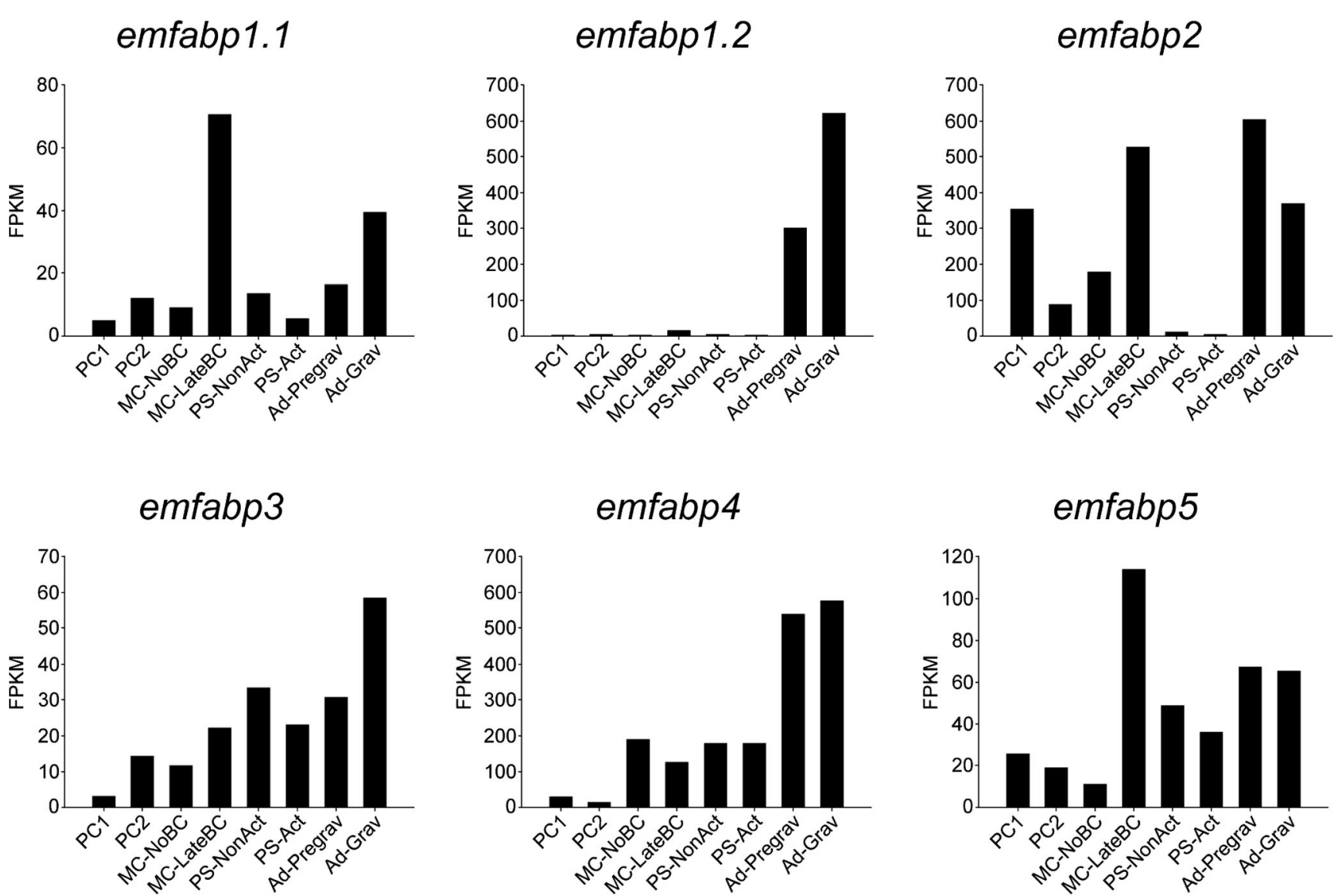

Fig. 4 Transcripts' level for the different EmFABP genes. The figure shows the transcription levels (in fragments per kilobase of exon model

2 days old; PC2, primary cells 11 days old; MC-noBC, metacestodes without brood capsules; MC-lateBC, late metacestode vesicles with brood capsules; PS-nonact, non-activated protoscoleces; PS-act, activated protoscoleces; Ad-pregrav, pregravid adult worms; Ad-grav, gravid adult E. multilocularis in different stages (or samples) of the parasite. All transcriptomic data were obtained from Tsai et al. (2013) and reanalysed as described in the "Materials and methods" section. PC1, primary cells

E. multilocularis reference genome from WormBase ParaSite (WBPS7) using the Hisat2 v2.0.5. Reads for each transcript (annotations from WormBase ParaSite and a manual annotation for emfabp5) were counted using the HTSeqCount v0.7.1 with a minimal quality score of 30 to filter out multiplemapped reads, allowing qualitative distinction between emfabp1.1 and emfabp1.2. As this method is not suitable to calculate realistic quantitative expression levels for genes with identical sequences, we used a second approach to estimate those. RNAseq data from (Tsai et al. 2013) and predicted E. multilocularis transcripts from WormBase ParaSite (WBPS7) together with the sequenced transcript for emfabp 5 were used to estimate expression levels with the Kallisto v0.43.1.

\section{Parasite material}

Parasite material was maintained in Mongolian jirds as described in Spiliotis et al. (2008) and Spiliotis and Brehm (2009) with approval from the ethics committee of the
Government of Lower Franconia (permit no. 55.2-2532-2354 ) according to German and European regulation on animal protection (Tierschutzgesetz). E. multilocularis primary cells were obtained from in vitro-cultured metacestodes, as previously described (Koziol et al. 2014).

\section{RT-PCR and cloning}

RNA from primary cell cultures ( 2 days cultures) was extracted employing Isol-RNA Lysis Reagent (5Prime) according to the manufacturer's instructions, and treated with DNase (RQ1 RNase-free DNase, Promega). cDNA was synthesised employing poli-dT oligonucleotides and PrimeScript Reverse Transcriptase (TaKaRa) as recommended by the manufacturer. The obtained cDNA was employed as a template for PCR amplification of the coding sequences of the predicted FABP genes. PCRs were performed employing Platinum Pfx DNA polymerase (Invitrogen) and the corresponding primers for each sequence: EmFABP1.1-CDS-Fw, atggaggcgttcctcggta; EmFABP1.1-CDS-Rv, 
ttacgacacctttgagtaggttc; EmFAB P 2-CDS-Fw, atggagccattcatcggta; Em F A B P 2-C D S - R v, ttacatccctcttgagtaggttcg; EmFABP3-CDS-Fw, atggatgactttctgggcacct; EmFABP3-CDS-Rv, tcagtcctttactcgacgataca; EmFABP4-CDS-Fw, atggatgaatttctgggatcctg; EmFABP4-CDS-Rv, ttatttgtcgaattagtattatccaa; EmFABP5-CDS-Fw, atggagg c a t tc ctagg c a c; E m F A B P 5 - C D S - R v, ctactccactcgtttgtaagtt. It is important to note that CDS sequence of EmFABP1.2 is exactly the same as EmFABP1.1. After purification, the amplified DNA fragments were Atailed and ligated to pGEM-T Easy vector (Promega), according to the protocol supplied by the manufacturer. The inserts of plasmids were sequenced at Macrogen, Inc. (Korea), employing SP6 promoter and T7 promoter universal primers. The obtained sequences were aligned to the predicted sequences employing the tool CLUSTALW and visual inspection.

\section{Sequence alignment and phylogenetic analysis}

In order to define the relatedness of the proteins across the species, a phylogenetic analysis was performed using cestode and model species FABPs. FABPs sequences were aligned using CLUSTALW; the sequences were adjusted with manual edition when needed. The phylogenetic tree was inferred using maximum likelihood method based on the JTTmatrixbased model. The bootstrap consensus tree was inferred from 500 replicates. Branches corresponding to partitions reproduced in less than $50 \%$ bootstrap replicates are collapsed. The analysis involved 72 amino acid sequences. There were a total of 117 of the final dataset. Evolutionary analysis was conducted in the Mega 7 (Kumar et al. 2016).

Funding information This work was supported by the National Research Council of Argentina (CONICET), the National Agency for Scientific and Technological Promotion (ANPCYT), the National University of La Plata (UNLP) and the European Molecular Biology Organization short term fellowship (grant number ASTF 344-2011 (to JLP)).

\section{Compliance with ethical standards}

Conflict of interests The authors declare that they have no conflict of interest.

\section{References}

Alvite G, Canclini L, Corvo I, Esteves A (2008) Two novel Mesocestoides vogae fatty acid binding proteins-functional and evolutionary implications. FEBS J 275:107-116. https://doi.org/ 10.1111/j.1742-4658.2007.06179.x

Alvite G, Di Pietro SM, Santomé JA et al (2001) Binding properties of Echinococcus granulosus fatty acid binding protein. Biochim Biophys Acta 1533:293-302
Esteves A, Dallagiovanna B, Ehrlich R (1993) A developmentally regulated gene of Echinococcus granulosus codes for a 15.5-kilodalton polypeptide related to fatty acid binding proteins. Mol Biochem Parasitol 58:215-222

Esteves A, Ehrlich R (2006) Invertebrate intracellular fatty acid binding proteins. Comp Biochem Physiol Part C 142:262-274. https://doi. org/10.1016/j.cbpc.2005.11.006

Esteves A, Portillo V, Ehrlich R (2003) Genomic structure and expression of a gene coding for a new fatty acid binding protein from Echinococcus granulosus. Biochim Biophys Acta Mol Cell Biol Lipids 1631:26-34. https://doi.org/10.1016/S1388-1981(02)003219

Haunerland NH, Spener F (2004) Fatty acid-binding proteins - insights from genetic manipulations. Prog Lipid Res 43:328-349. https://doi. org/10.1016/j.plipres.2004.05.001

Huang F, Dang Z, Suzuki Y, Horiuchi T, Yagi K, Kouguchi H, Irie T, Kim $\mathrm{K}$, Oku Y (2016) Analysis on gene expression profile in oncospheres and early stage metacestodes from Echinococcus multilocularis. PLoS Negl Trop Dis 10:e0004634. https://doi.org/ 10.1371/journal.pntd.0004634

Jakobsson E, Alvite G, Bergfors T, Esteves A, Kleywegt GJ (2003) The crystal structure of Echinococcus granulosus fatty-acid-binding protein 1. Biochim Biophys Acta 1649:40-50. https://doi.org/10.1016/ S1570-9639(03)00151-1

Jones TA, Bergfors T, Sedzik J, Unge T (1988) The three-dimensional structure of P2 myelin protein. EMBO J 7:1597-1604

Koziol U, Rauschendorfer T, Zanon Rodríguez L et al (2014) The unique stem cell system of the immortal larva of the human parasite Echinococcus multilocularis. Evodevo 5:1-23. https://doi.org/10. 1186/2041-9139-5-10

Kumar S, Stecher G, Tamura K (2016) MEGA7: molecular evolutionary genetics analysis version 7.0 for bigger datasets. Mol Biol Evol 33: 1870-1874. https://doi.org/10.1093/molbev/msw054

Maule AG, Marks NJ (eds) (2006) Parasitic flatworms: molecular biology, biochemistry, immunology and physiology. CABI

Morphew RM, Wilkinson TJ, MacKintosh N et al (2016) Exploring and expanding the fatty-acid-binding protein superfamily in Fasciola species. J Proteome Res 15:3308-3321. https://doi.org/10.1021/ acs.jproteome.6b00331

Parada GE, Munita R, Cerda CA, Gysling K (2014) A comprehensive survey of non-canonical splice sites in the human transcriptome. Nucleic Acids Res 42:1-15. https://doi.org/10.1093/nar/gku744

Pórfido JL, Alvite G, Silva V et al (2012) Direct interaction between EgFABP1, a fatty acid binding protein from Echinococcus granulosus, and phospholipid membranes. PLoS Negl Trop Dis 6: e1893

Sheth N, Roca X, Hastings ML, Roeder T, Krainer AR, Sachidanandam $\mathrm{R}$ (2006) Comprehensive splice-site analysis using comparative genomics. Nucleic Acids Res 34:3955-3967. https://doi.org/10.1093/ nar/gk1556

Smathers RL, Petersen DR (2011) The human fatty acid-binding protein family: evolutionary divergences and functions. Hum Genomics 5: 170-191

Smyth JD, McManus DP (2007) The physiology and biochemistry of cestodes. Cambridge University Press

Spiliotis M, Brehm K (2009) Axenic in vitro cultivation of Echinococcus multilocularis metacestode vesicles and the generation of primary cell cultures. Methods Mol Biol 470:245-262. https://doi.org/10. 1007/978-1-59745-204-5_17

Spiliotis M, Lechner S, Tappe D (2008) Transient transfection of Echinococcus multilocularis primary cells and complete in vitro regeneration of metacestode vesicles. Int J Parasitol 38:1025-1039. https://doi.org/10.1016/j.ijpara.2007.11.002

Tsai IJ, Zarowiecki M, Holroyd N, Garciarrubio A, Sánchez-Flores A, Brooks KL, Tracey A, Bobes RJ, Fragoso G, Sciutto E, Aslett M, Beasley H, Bennett HM, Cai X, Camicia F, Clark R, Cucher M, de 
Silva N, Day TA, Deplazes P, Estrada K, Fernández C, Holland PWH, Hou J, Hu S, Huckvale T, Hung SS, Kamenetzky L, Keane JA, Kiss F, Koziol U, Lambert O, Liu K, Luo X, Luo Y, Macchiaroli N, Nichol S, Paps J, Parkinson J, Pouchkina-Stantcheva N, Riddiford N, Rosenzvit M, Salinas G, Wasmuth JD, Zamanian M, Zheng Y, Taenia solium Genome Consortium, Cai J, Soberón X, Olson PD, Laclette JP, Brehm K, Berriman M (2013) The genomes of four tapeworm species reveal adaptations to parasitism. Nature 496:57-63. https://doi.org/10.1038/nature12031

World Health Organization (2019) https://www.who.int/news-room/factsheets/detail/echinococcosis. Accessed 15 May 2019

Xu M, Joo H-J, Paik Y-K (2011) Novel functions of lipid-binding protein 5 in Caenorhabditis elegans fat metabolism. J Biol Chem 286: 28111-28118. https://doi.org/10.1074/jbc.M111.227165
Zheng H, Zhang W, Zhang L et al (2013a) The genome of the hydatid tapeworm Echinococcus granulosus. Nat Genet 45:1168-1177. https://doi.org/10.1038/ng.2757

Zheng Y, Blair D, Bradley JE (2013b) Phyletic distribution of fatty acidbinding protein genes. PLoS One 8:e77636. https://doi.org/10.1371/ journal.pone.0077636

Zimmerman AW, Veerkamp JH (2002) New insights into the structure and function of fatty acid-binding proteins. Cell Mol Life Sci 59: 1096-1116. https://doi.org/10.1007/s00018-002-8490-y

Publisher's note Springer Nature remains neutral with regard to jurisdictional claims in published maps and institutional affiliations. 